\title{
PRIMITIVE DIGRAPHS WITH LARGE EXPONENTS AND SLOWLY SYNCHRONIZING AUTOMATA
}

\author{
D. S. Ananichev, ${ }^{*}$ M. V. Volkov, ${ }^{*}$ and V. V. Gusev*
}

UDC 519.713.4+519.172.3

We present several infinite series of synchronizing automata for which the minimum length of reset words is close to the square of the number of states. All these automata are tightly related to primitive digraphs with large exponents. Bibliography: 28 titles.

\section{BACKGROUND AND THE STRUCTURE OF THE PAPER}

This paper has arisen from our attempts to find a theoretical explanation for the results of certain computational experiments in synchronization of finite automata. Recall that a (complete deterministic) finite automaton (DFA) is a triple $\mathscr{A}=\langle Q, \Sigma, \delta\rangle$ where $Q$ and $\Sigma$ are finite sets called the state set and the input alphabet, respectively, and $\delta: Q \times \Sigma \rightarrow Q$ is a totally defined function called the transition function. As usual, $\Sigma^{*}$ stands for the collection of all finite words over the alphabet $\Sigma$, including the empty word 1 . The function $\delta$ extends to a function $Q \times \Sigma^{*} \rightarrow Q$ (still denoted by $\delta$ ) in the following natural way: for every $q \in Q$ and $w \in \Sigma^{*}$, we set $\delta(q, w)=q$ if $w=1$ and $\delta(q, w)=\delta(\delta(q, v), a)$ if $w=v a$ for some $v \in \Sigma^{*}$ and $a \in \Sigma$. Thus, via $\delta$, every word $w \in \Sigma^{*}$ acts on the set $Q$.

A DFA $\mathscr{A}=\langle Q, \Sigma, \delta\rangle$ is said to be synchronizing if some word $w \in \Sigma^{*}$ brings all states to one particular state: $\delta(q, w)=\delta\left(q^{\prime}, w\right)$ for all $q, q^{\prime} \in Q$. Any such word $w$ is said to be a reset word for the DFA. The minimum length of reset words for $\mathscr{A}$ is called the reset threshold of $\mathscr{A}$.

Synchronizing automata serve as transparent and natural models of error-resistant systems in many applied areas (system and protocol testing, information encoding, robotics). At the same time, synchronizing automata surprisingly arise in some parts of pure mathematics (symbolic dynamics, the theory of substitution systems, and others). The basics of the theory of synchronizing automata, as well as its diverse connections and applications, are discussed, for instance, in the recent surveys [19,26]. Here we focus on only one aspect of the theory, namely, on the question of how the reset threshold of a DFA depends on the number of states.

For brevity, a DFA with $n$ states will be referred to as an n-automaton. In 1964, Černý [9] constructed a series of synchronizing $n$-automata with reset threshold $(n-1)^{2}$. Soon after that, he conjectured that these automata represent the worst possible case with respect to synchronization speed, i.e., that every synchronizing $n$-automaton can be reset by a word of length $(n-1)^{2}$. This hypothesis became known as the Černý conjecture. In spite of its simple formulation and efforts of many researchers, the Černý conjecture remains unproved (and undisproved) for more than 45 years. Moreover, no upper bound of magnitude $O\left(n^{2}\right)$ for the reset threshold of a synchronizing $n$-automaton is known so far. ${ }^{1}$

Why is the Černý conjecture so inaccessible? A detailed discussion of this important issue would go far beyond the scope of the present paper, but one of the difficulties encountered by the theory of synchronizing automata is worth registering here. We mean a shortage of examples of extremal automata, i.e., $n$-automata having reset threshold $(n-1)^{2}$. In fact, the series found in [9] still remains the only known infinite series of extremal automata. Besides that, we know only a few isolated examples of such automata, the largest one (with respect to the number of states) being the 6-automaton discovered by Kari [13] in 2001. (See [26] for a complete list of known extremal automata.) Moreover, even $n$-automata whose reset threshold is close to $(n-1)^{2}$ have been very rare in the literature so far - besides the Cerný series one can only refer to the series from [4]. With a very restricted number of examples, it was difficult to verify various guesses and assumptions that arose when researchers were searching for approaches to the Černý conjecture. That is why the history of investigations in this area abounds in "false trails," i.e., auxiliary conjectures that looked promising at first but were disproved after some time. (See [6] for an analysis of a number of such "false trails.")

\footnotetext{
*Institute of Mathematics and Computer Science, Ural Federal University, Ekaterinburg, Russia, e-mail: Dmitry.Ananichev@usu.ru, Mikhail.Volkov@usu.ru, vl.gusev@gmail.com.

${ }^{1} \mathrm{Up}$ to now, the best upper bound on the reset threshold of a synchronizing $n$-automaton is the bound $\frac{n^{3}-n}{6}$ found by Pin [16] in 1983. The slightly better upper bound $\frac{n\left(7 n^{2}+6 n-16\right)}{48}$ has been recently published in [25], but the proof of this result contains an unclear step.
}

Translated from Zapiski Nauchnykh Seminarov POMI, Vol. 402, 2012, pp. 9-39. Original article submitted December $23,2011$. 
How can one find slowly synchronizing automata? Experiments (see, e.g., [20]) demonstrate that with probability very close to 1 , a random automaton is reset by a word of length much less than the number of states. Therefore, it is impossible to encounter by chance an automaton whose reset threshold is close to the square of the number of states, and one has to reveal such automata via exhaustive search. It was such an exhaustive search experiment that served as a departure point of the present paper.

Our methodology and some results of the experiment are described in Sec. 2. We have noticed a similarity between the observable behavior of the number of synchronizing automata with a fixed number of states as a function of their reset threshold and the well-studied behavior of the number of primitive digraphs with a fixed number of vertices as a function of their exponent. We discuss this similarity in Sec. 3 after recalling the necessary concepts and facts from the theory of primitive digraphs. The main results of the paper are collected in Sec. 4. We show that slowly synchronizing automata revealed in our experiment represent some infinite series of such automata and that each of these series is tightly related to a certain known series of primitive digraphs with large exponent. This connection between digraphs and automata allows us to provide transparent and uniform proofs for all statements concerning the reset threshold of series of slowly synchronizing automata, both for the previously known series and the series that have first appeared in the present paper. The proof technique is, to the best of our knowledge, new and appears to be of independent interest. In Sec. 5, we discuss further prospects of the suggested approach and formulate a few new conjectures.

A preliminary version of a part of the results of this paper was published in [3].

\section{Methodology And some Results of the EXPERiment}

As mentioned in Sec. 1, finding automata whose reset threshold is close to the square of the number of states requires an exhaustive search. Since the number of $n$-automata grows drastically with $n$, such a search should be designed in a reasonable way. For instance, specifying a 9-automaton with two input letters is equivalent to specifying a pair of functions on a 9-element set. There are $9^{18} \approx 1.50 \times 10^{17}$ such pairs, and if one will spend one nanosecond on calculating the reset threshold of each automaton defined this way, the exhaustive search would take around five years. Clearly, if an $n$-automaton with $k$ input letters is specified by a $k$-tuple of functions on an $n$-element set, each particular automaton is generated $n ! k$ ! times. However, one cannot speed up the search by screening out isomorphic automata: even for $n=9$ and $k=2$, neither time nor memory would suffice to check whether the current automaton is isomorphic to one of the previously generated automata.

In order to optimize the search, we have employed the string representation of initially-connected automata suggested in [2]. Recall that a DFA $\mathscr{A}=\langle Q, \Sigma, \delta\rangle$ is said to be initially-connected (from a state $q_{0}$ ) if one can reach any state from $q_{0}$ by applying a suitable word: for every $q \in Q$ there exists $w \in \Sigma^{*}$ such that $q=\delta\left(q_{0}, w\right)$. A DFA that is initially-connected from each of its states is called strongly connected. In general, a synchronizing automaton may fail to be strongly connected or initially-connected. However, it is well known that one can restrict attention to strongly connected automata when dealing with issues related to the Černý conjecture. This is a consequence of the following easy result.

Proposition 1 ( [27, Proposition 2.1]). Let $\mathscr{A}=\langle Q, \Sigma, \delta\rangle$ be a synchronizing automaton, and let $S$ be the set of all states to which $\mathscr{A}$ can be reset. Then $\mathscr{S}=\left\langle S, \Sigma,\left.\delta\right|_{S}\right\rangle$ is a strongly connected subautomaton in $\mathscr{A}$, and for every function $f: \mathbb{Z}^{+} \rightarrow \mathbb{N}$ satisfying

$$
f(n) \geq \frac{n(n-1)}{2} \text { and } f(n) \geq f(n-m+1)+f(m) \text { for } n \geq m \geq 1,
$$

the fact that the reset threshold of the automaton $\mathscr{S}$ is bounded by $f(|S|)$ implies that the reset threshold of $\mathscr{A}$ does not exceed $f(|Q|)$.

In particular, taking $(n-1)^{2}$ as $f(n)$, we can conclude that if $\mathscr{A}$ is a counterexample for the Černý conjecture, then so is the strongly connected subautomaton $\mathscr{S}$. Similarly, if $\mathscr{A}$ has reset threshold close to the square of the number of the states, then so does $\mathscr{S}$. Thus, restricting our search to initially-connected automata, we do not risk to overlook a counterexample for the Černý conjecture or any interesting slowly synchronizing automaton.

Now we describe the string representation from [2]. Let a DFA $\mathscr{A}=\langle Q, \Sigma, \delta\rangle$ be initially-connected from a state $q_{0}$. We fix a linear ordering of the input alphabet $\Sigma$ and traverse the DFA by breadth-first search starting at $q_{0}$ and choosing the outgoing transitions according to the ordering. Let the state $q_{0}$ have number 0 , and let all other states in $Q$ be numbered in the order of their appearance in the breadth-first search. For instance, 
the states of the DFA in Fig. 1 are numbered as \begin{tabular}{c|c|c|c}
$A$ & $B$ & $C$ & $D$ \\
\hline 0 & 2 & 1 & 3
\end{tabular} provided that the input letters are ordered as $a<b<c$ and the state $A$ is chosen as $q_{0}$.

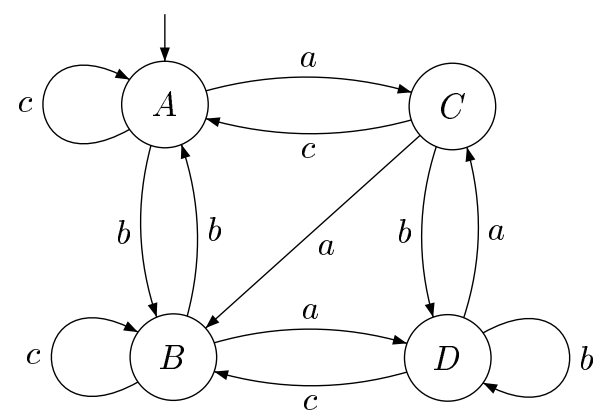

Fig. 1. The DFA with the canonical string $[1,2,0,2,3,0,3,0,2,1,3,2]$.

We assign a string of length $|\Sigma|$ to each state $q$ of $\mathscr{A}$; the $i$ th position of the string holds the number of the state to which $q$ is sent under the action of the $i$ th letter. If we concatenate all these strings in the increasing order of the state numbers, we get a string of numbers from the set $\{0,1, \ldots,|Q|-1\}$ that has length $|Q||\Sigma|$ and uniquely determines $\mathscr{A}$. It is called the canonical string of the DFA $\mathscr{A}$. For instance, the canonical string of the DFA in Fig. 1 is $[1,2,0,2,3,0,3,0,2,1,3,2]$.

It is not hard to see (cf. [2, Theorem 5]) that a string $\left[s_{0}, \ldots, s_{n k-1}\right]$ of numbers from the set $\{0,1, \ldots, n-1\}$ is the canonical string of an initially-connected $n$-automaton with $k$ input letters if and only if the following two conditions are satisfied:

(R1) for every $i$ such that $s_{i}>1$, there is $j<i$ with $s_{j}=s_{i}-1$,

(R2) for every $m$ such that $1 \leq m<n$, there is $j<m k$ with $s_{j}=m$.

Using this observation, we calculated the reset thresholds of initially-connected $n$-automata with two input letters as follows. We used a 128-core grid of AMD Opteron $2.6 \mathrm{GHz}$ processors. The grid belongs to the Institute of Mathematics and Mechanics of the Ural Branch of the Russian Academy of Sciences; it runs under Linux, has $256 \mathrm{~Gb}$ of memory and the peak performance of 665.6 GFLOPS. One node of the gird generated relatively small portions of strings satisfying (R1) and (R2) and sent them to other nodes that worked on their portions of automata in parallel. The management program was written in C with MPI. Standard algorithms (cf. [19, 26]) were implemented to test whether the current automaton is synchronizing and to calculate its reset threshold. Both implementations were written in C. Note that the synchronization test is very fast, since it works with the digraph of pairs of states, while the calculation of the reset threshold works with the digraph of nonempty sets of states and in the worst case its running time depends exponentially on the size of the automaton under inspection. ${ }^{2}$ However, in practice the reset threshold was calculated fairly fast, since, as mentioned in Sec. 1, it is small for an overwhelming majority of automata.

Presenting automata via their canonical strings drastically reduces the exhaustive search. (It is easy to see, for instance, that every $n$-automaton with two input letters is generated at most $2 n$ times if presented this way.) Nevertheless, the search still remains quite large. For $n=9$, say, the number of automata to be analyzed was 705 068085303 . However, thanks to parallelization, the computation for $n=9$ took less than 24 hours.

As the result of the computation, we built an array that, for each possible value of reset threshold, contains the number of automata attaining this value. A part of results that was of outmost importance for us (namely, the part related to slowly synchronizing automata) had been double-checked with the package TESTAS [22].

Table 1 shows a part of the output array for the case $n=9$. Here automata are counted up to isomorphism.

Clearly, the unique automaton in the column corresponding to $N=64$ is nothing but the 9-automaton from the Černý series. Then one observes a gap: no 9-automata with two input letters have reset threshold in the range from 59 to 63 . This gap was mentioned by Trahtman [22,23], who reported that for $n=7,8,9,10$ no $n$-automata with two input letters and reset threshold in the range from $n^{2}-3 n+5$ to $n^{2}-2 n$ had been registered

\footnotetext{
${ }^{2}$ It is known ([15, Theorem 4]) that the problem of computing the reset threshold of a given automaton is complete for the functional analog $\mathrm{FP}^{\mathrm{NP}[\mathrm{log}]}$ of the complexity class $\mathrm{P}^{\mathrm{NP}[\mathrm{log}]}$ consisting of all decision problems solvable by a deterministic polynomialtime Turing machine that has access to an oracle for an NP-complete problem, with the number of queries being logarithmic in the size of the input.
} 
in his experiments. The gap is followed by an "island" consisting of three values attained by 6 automata, and the "island" is followed by yet another gap. To the best of our knowledge, the second gap has not been reported in the literature up to now.

Table 1. The reset thresholds of synchronizing 9-automata with two input letters.

\begin{tabular}{|l||c|c|c|c|c|c|c|c|c|c|c|c|c|c|}
\hline \multicolumn{1}{|c||}{$N$} & 64 & 63 & 62 & 61 & 60 & 59 & 58 & 57 & 56 & 55 & 54 & 53 & 52 & 51 \\
\hline $\begin{array}{l}\text { \# of automata } \\
\text { with reset } \\
\text { threshold } N\end{array}$ & 1 & 0 & 0 & 0 & 0 & 0 & 1 & 2 & 3 & 0 & 0 & 0 & 4 & 4 \\
\hline
\end{tabular}

The behavior just described - a unique extremal value followed by a gap which in turn is followed by a small "island" and yet another gap - persists also for automata with a larger number of states. The size of the "island" depends only on the parity of the number of states, and the sizes of the gaps grow as linear functions of the number of states. A similar behavior is known for another value investigated in discrete mathematics, namely, for the number of primitive digraphs with a fixed number of vertices and a given exponent. In the next section, we recall these notions and discuss the similarity in more detail.

\section{Primitive Digraphs AND THEIR EXPONENTS}

A digraph (directed graph) is a pair $D=\langle V, E\rangle$ where $V$ is a finite set and $E \subseteq V \times V$. The elements of $V$ and $E$ are called vertices and edges, respectively. Note that this definition allows loops but excludes multiple edges. If $v, v^{\prime} \in V$ and $e=\left(v, v^{\prime}\right) \in E$, then we say that the edge $e$ starts at $v$. We assume that the reader is acquainted with the basic notions of digraph theory, such as directed path, directed cycle, isomorphism, etc.

If $D=\langle V, E\rangle$ is a digraph, then its incidence matrix (referred to simply as its matrix in what follows) is a $V \times V$ matrix whose entry in row $v$ and column $v^{\prime}$ is 1 if $\left(v, v^{\prime}\right) \in E$ and 0 otherwise. Conversely, to each $n \times n$ matrix $P=\left(p_{i j}\right)$ with nonnegative real entries one can assign a digraph $D(P)$ on the vertex set $\{1,2, \ldots, n\}$ in which a pair $(i, j)$ is an edge if and only if $p_{i j}>0$. This correspondence between matrices and digraphs allows one to state in the language of digraphs a number of important notions and results from the classical theory of nonnegative matrices (the Perron-Frobenius theory).

A digraph $D=\langle V, E\rangle$ is said to be strongly connected if for every pair $\left(v, v^{\prime}\right) \in V \times V$ there is a directed path from $v$ to $v^{\prime}$. A strongly connected digrpah $D$ is called primitive if the greatest common divisor of the lengths of the directed cycles of $D$ is equal to 1 . In the literature, such digraphs are sometimes called aperiodic. Our choice of the name is justified by the fact that a digraph has this property if and only if its matrix is primitive in the sense of the Perron-Frobenius theory, that is, the matrix has a positive eigenvalue that is strictly greater than the absolute value of any of its other eigenvalues.

The $t$ th power of a digraph $D=\langle V, E\rangle$ is the digraph $D^{t}$ with the same vertex set $V$ in which a pair $\left(v, v^{\prime}\right) \in V \times V$ is an edge if and only if $D$ has a directed path from $v$ to $v^{\prime}$ of length precisely $t$. It is easy to see that if $M$ is the matrix of $D$, then the digraph $D^{t}$ is isomorphic to the digraph $D\left(M^{t}\right)$, where $M^{t}$ is the usual $t$ th power of the matrix $M$. It is known (see, e.g., [18, p. 224]) that if $D$ is a primitive digraph, then for some $t$ the power $D^{t}$ is a complete digraph (with loops), that is, in $D^{t}$ each pair of vertices constitutes an edge. In matrix terms, this means that each entry of the matrix $M^{t}$ is positive. The least $t$ with this property is called the exponent of the digraph $D$ and is denoted by $\gamma(D)$. Exponents of digraphs have been intensively studied over the last 60 years, and we refer to [7] for a survey of results accumulated in this area. In this paper, we need only a few classical results collected in the following theorem. For brevity, in this theorem (and in the rest of the paper) a digraph with $n$ vertices is called an $n$-digraph.

Theorem 1. (a) (Wielandt's theorem, see $[10,11,28]$ ). If $D$ is a primitive $n$-digraph, then $\gamma(D) \leq(n-1)^{2}+1$.

(b) ([11, Theorem 6 and Corollary 4]). If $n>2$, then, up to isomorphism, there is exactly one primitive $n$-digraph $D$ with $\gamma(D)=(n-1)^{2}+1$, and exactly one primitive $n$-digraph $D$ with $\gamma(D)=(n-1)^{2}$. The matrices of these digraphs are

$$
\left(\begin{array}{cccccc}
0 & 1 & 0 & \ldots & 0 & 0 \\
0 & 0 & 1 & \ldots & 0 & 0 \\
\ldots & \ldots & \ldots & \ldots & \ldots & \ldots \\
0 & 0 & 0 & \ldots & 0 & 1 \\
1 & 1 & 0 & \ldots & 0 & 0
\end{array}\right) \text { and }\left(\begin{array}{cccccc}
0 & 1 & 0 & \ldots & 0 & 0 \\
0 & 0 & 1 & \ldots & 0 & 0 \\
\ldots & \ldots & \ldots & \ldots & \ldots & \ldots \\
1 & 0 & 0 & \ldots & 0 & 1 \\
1 & 1 & 0 & \ldots & 0 & 0
\end{array}\right) \text {, respectively. }
$$


(c) $\left(\left[11\right.\right.$, Theorem 7]). If $n>4$ is even, then there is no primitive $n$-digraph $D$ such that $n^{2}-4 n+6<\gamma(D)<$ $(n-1)^{2}$, and, up to isomorphism, there are either 3 or 4 primitive $n$-digraphs $D$ with $\gamma(D)=n^{2}-4 n+6$, according to wheter or not $n$ is a multiple of 3 .

(d) $\left(\left[11\right.\right.$, Theorem 8]). If $n>3$ is odd, then there is no primitive $n$-digraph $D$ such that $n^{2}-3 n+4<\gamma(D)<$ $(n-1)^{2}$, and, up to isomorphism, there is exactly one primitive $n$-digraph $D$ with $\gamma(D)=n^{2}-3 n+4$, exactly one with $\gamma(D)=n^{2}-3 n+3$, and exactly two with $\gamma(D)=n^{2}-3 n+2$. The matrices of these digraphs are

$$
\begin{aligned}
& \left(\begin{array}{cccccc}
0 & 1 & 0 & \ldots & 0 & 0 \\
0 & 0 & 1 & \ldots & 0 & 0 \\
\ldots & \ldots & \ldots & \ldots & \ldots & \ldots \\
0 & 0 & 0 & \ldots & 1 & 0 \\
0 & 0 & 0 & \ldots & 0 & 1 \\
1 & 0 & 1 & \ldots & 0 & 0
\end{array}\right), \quad\left(\begin{array}{cccccc}
0 & 1 & 0 & \ldots & 0 & 0 \\
0 & 0 & 1 & \ldots & 0 & 0 \\
\ldots & \ldots & \ldots & \ldots & \ldots \\
0 & 0 & 0 & \ldots & 1 & 0 \\
0 & 1 & 0 & \ldots & 0 & 1 \\
1 & 0 & 1 & \ldots & 0 & 0
\end{array}\right), \\
& \left(\begin{array}{cccccc}
0 & 1 & 0 & \ldots & 0 & 0 \\
0 & 0 & 1 & \ldots & 0 & 0 \\
\ldots & \ldots & \ldots & \ldots & \ldots \\
1 & 0 & 0 & \ldots & 1 & 0 \\
0 & 1 & 0 & \ldots & 0 & 1 \\
1 & 0 & 1 & \ldots & 0 & 0
\end{array}\right),
\end{aligned}
$$

(e) ([11, Theorem 8]). If $n>3$ is odd, then there is no primitive $n$-digraph $D$ such that $n^{2}-4 n+6<\gamma(D)<$ $n^{2}-3 n+2$, and, up to isomorphism, there are either 3 or 4 primitive $n$-digraphs $D$ with $\gamma(D)=n^{2}-4 n+6$, according to whether or not $n$ is a multiple of 3.

In Table 2, we compare the experimental data from Table 1 with the data that we can extract from Theorem 1. Both digraphs and automata are counted up to isomorphism.

Table 2. The exponents of primitive 9-digraphs with 9 vertices vs. the reset thresholds for 2-letter synchronizing automata with 9 states.

\begin{tabular}{|l||c|c|c|c|c|c|c|c|c|c|c|c|c|c|c|}
\hline \multicolumn{1}{|c|}{$N$} & 65 & 64 & 63 & 62 & 61 & 60 & 59 & 58 & 57 & 56 & 55 & 54 & 53 & 52 & 51 \\
\hline $\begin{array}{l}\text { \# of digraphs } \\
\text { with exponent } N\end{array}$ & 1 & 1 & 0 & 0 & 0 & 0 & 0 & 1 & 1 & 2 & 0 & 0 & 0 & 0 & 3 \\
\hline $\begin{array}{l}\text { \# of automata } \\
\text { with reset } \\
\text { threshold } N\end{array}$ & 0 & 1 & 0 & 0 & 0 & 0 & 0 & 1 & 2 & 3 & 0 & 0 & 0 & 4 & 4 \\
\hline
\end{tabular}

There is an obvious similarity between the second and the third rows of Table 2. An analogous similarity is revealed when one compares the data for other sizes of automata/digraphs. We believe that the observed similarity is more than a mere coincidence and that, on the contrary, it reflects some profound and perhaps yet hidden interconnections between primitive digraphs and synchronizing automata. Some of such interconnections were discovered in the course of investigations related to the so-called road coloring problem. We recall the notions involved there.
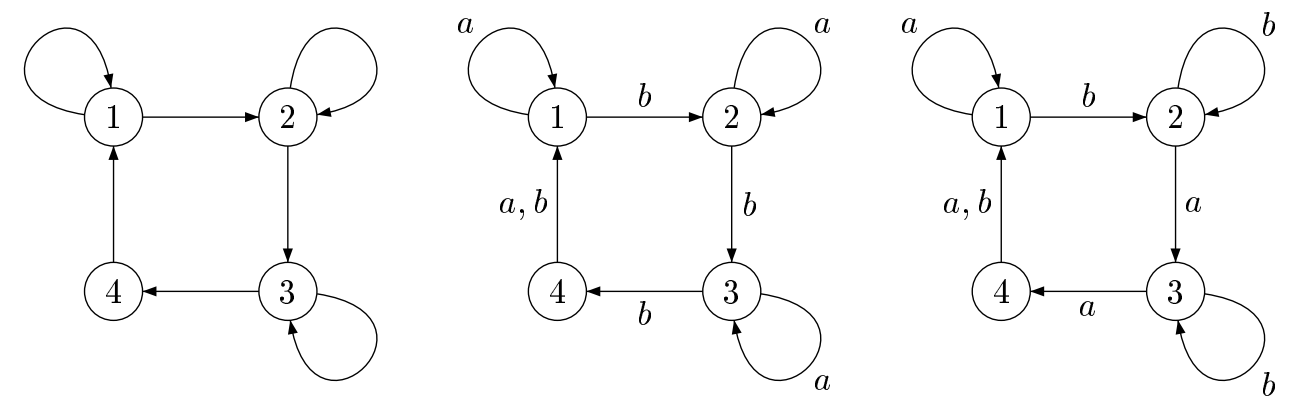

Fig. 2. A digraph and two of its colorings. 
Given a DFA $\mathscr{A}=\langle Q, \Sigma, \delta\rangle$, its digraph $D(\mathscr{A})$ has $Q$ as the vertex set, and $\left(q, q^{\prime}\right) \in Q \times Q$ is an edge of $D(\mathscr{A})$ if and only if $q^{\prime}=\delta(q, a)$ for some $a \in \Sigma$. It is easy to see that a digraph $D$ is isomorphic to the digraph of some DFA if and only if each vertex of $D$ has at least one outgoing edge. In what follows, we always consider only digraphs satisfying this property. Every DFA $\mathscr{A}$ such that $D \cong D(\mathscr{A})$ is called a coloring of $D$. Thus every coloring of $D$ is defined by assigning nonempty sets of labels (colors) from some alphabet $\Sigma$ to the edges of $D$ so that the label sets assigned to the outgoing edges of each vertex form a partition of $\Sigma$. Figure 2 shows a digraph and two of its colorings by $\Sigma=\{a, b\}$.

In 1977, Adler, Goodwyn, and Weiss [1] observed that the digraph of every strongly connected synchronizing automaton is primitive and conjectured that every primitive digraph has a synchronizing coloring. This conjecture, known as the road coloring conjecture, was recently proved by Trahtman [24]. It is easy to relate the reset threshold of a strongly connected synchronizing automaton to the exponent of its digraph.

Proposition 2. Let $\mathscr{A}=\langle Q, \Sigma, \delta\rangle$ be a strongly connected synchronizing n-automaton with reset threshold $t$. Then

$$
\gamma(D(\mathscr{A})) \leq t+n-1 .
$$

Proof. Let $w \in \Sigma^{*}$ be a reset word of length $t$, and let $p$ be such that $\delta(q, w)=p$ for each $q \in Q$. Now we take an arbitrary pair $\left(q^{\prime}, q^{\prime \prime}\right) \in Q \times Q$ and construct a directed path from $q^{\prime}$ to $q^{\prime \prime}$ of length precisely $t+n-1$ in the digraph $D(\mathscr{A})$. Since the digraph $D(\mathscr{A})$ is strongly connected, it has a directed path from $p$ to $q^{\prime \prime}$. Let $\ell$ be the minimum length of such a path. Since a path of minimum length visits no vertex twice, $\ell \leq n-1$. Now we consider an arbitrary directed path of length $n-1-\ell$ starting at $q^{\prime}$. From the endpoint of this path, we walk along the directed path of length $t$ labeled by the word $w$ in the automaton $\mathscr{A}$. Since $w$ is a reset word, this path necessarily ends with $p$. It remains to walk along the directed path of length $\ell$ from $p$ to $q^{\prime \prime}$ in order to obtain a directed path from $q^{\prime}$ to $q^{\prime \prime}$ of length $(n-1-\ell)+t+\ell=t+n-1$.

Thus colorings of primitive digraphs with large exponents yield automata with large reset thresholds. This observation is not yet sufficient to completely explain the similarity between the rows of Table 2, since many automata corresponding to nonzero entries in the third row cannot be obtained as colorings of primitive digraphs with large exponents. In Sec. 4, we present yet another way to produce slowly synchronizing automata from primitive digraphs.

\section{SERIES OF SLOWLY SYNCHRONIZING AUTOMATA}

4.1. Overview. First of all, we would like to discuss what kind of automata we are interested in; in other words, what is the precise meaning of expressions like "slowly synchronizing automata" or "automata whose reset threshold is close to the square of the number of states." As mentioned in Sec. 1, the reset threshold of an $n$-automaton can reach $(n-1)^{2}$. The corresponding example (discovered by Černý [9]) is the automaton $\mathscr{C}_{n}=\langle\{1,2, \ldots, n\},\{a, b\}, \delta\rangle$ where the letters $a$ and $b$ act as follows:

$$
\delta(i, a)=\left\{\begin{array}{ll}
i & \text { if } i<n, \\
1 & \text { if } i=n ;
\end{array} \quad \delta(i, b)= \begin{cases}i+1 & \text { if } i<n, \\
1 & \text { if } i=n .\end{cases}\right.
$$

The automaton $\mathscr{C}_{n}$ is shown in Fig. 5 (left). It is easy to see that if one adds a new state $q_{0}$ to the automaton $\mathscr{C}_{n-1}$ and then defines the action of the letters $a$ and $b$ at this added state in all possible ways such that at least one of the letters does not fix $q_{0}$, then one gets $n^{2}-1$ nonisomorphic initially-connected $n$-automata with reset thresholds between $(n-2)^{2}$ and $(n-2)^{2}+1$. In a similar way one can "multiply" other $(n-1)$-automata whose reset threshold is close to $(n-2)^{2}$, thus obtaining families of $n$-automata.

Since we want to avoid considering such more or less trivial modifications, we focus on $n$-automata whose reset thresholds are between $(n-2)^{2}+2$ and $(n-1)^{2}$. (This explains, in particular, our choice of the range of reset thresholds in Tables 1 and 2.)

Our experiments show that the number of synchronizing automata in this range is not large and that their distribution with respect to the possible values of reset threshold clearly reveals the following pattern: an isolated extreme value - a gap - a small "island" - another gap - a "continent," see Table 1 and the discussion at the end of Sec. 2. We will show that departing from the primitive digraphs with large exponents presented in Theorem 1, the following series of automata over a 2-letter alphabet can be constructed:

- the series $\mathscr{C}_{n}$ that corresponds to the observed extreme value; 
- the series $\mathscr{W}_{n}, \mathscr{D}_{n}^{\prime}, \mathscr{D}_{n}^{\prime \prime}, \mathscr{E}_{n}$, and for odd $n$ also the series $\mathscr{B}_{n}$ and $\mathscr{F}_{n}$, that correspond to all observed "island" values;

- the series $\mathscr{G}_{n}$ (for odd $n$ ) and $\mathscr{H}_{n}$ that correspond to the maximum observed "continental" values.

Figure 3 demonstrates which series of digraphs give rise to the series of automata just listed. (For clarity, digraph series in Fig. 3 are presented by their "icons" rather than matrices from Theorem 1.) A solid arrow from an icon to a letter denoting a series of automata means that the automata in the series are colorings of the corresponding digraphs; a dotted arrow indicates another way of producing automata from digraphs. Dotted frames embrace series of automata with the same value of reset threshold.

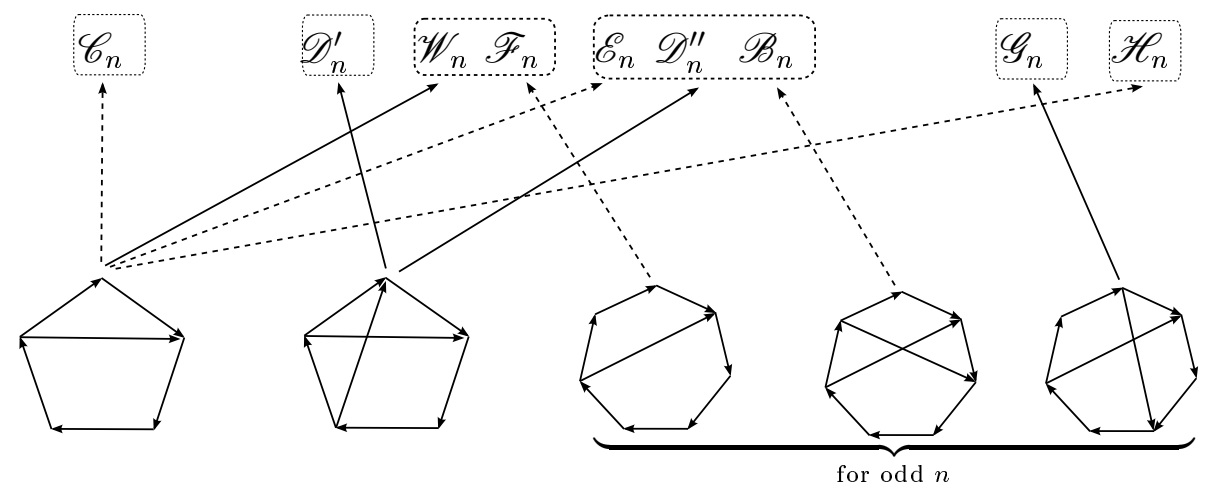

Fig. 3. Connections between series of primitive digraphs with large exponents and series of slowly synchronizing automata.

Thus we are going to establish a number of results about the series of automata listed in Fig. 3. (Two of these results - those concerning the series $\mathscr{C}_{n}$ and $\mathscr{B}_{n}$ - are known in the literature, but our proofs are essentially novel.) The results are divided into 5 groups according to the "origin" of the series, that is, according to the type of digraphs that give rise to automata in the series.

4.2. Automata related to digraphs of the series $W_{n}$. The digraph $W_{n}$ is the $n$-digraph with largest exponent that corresponds to the first matrix in (1). If we denote the vertices of $W_{n}$ by $1,2, \ldots, n$, then its edges are $(n, 1),(n, 2)$, and $(i, i+1)$ for $i=1, \ldots, n-1$. It is easy to see that, up to isomorphism and renaming of letters, there is a unique coloring of the digraph $W_{n}$ with two letters. We denote the resulting automaton by $\mathscr{W}_{n}$. The digraph $W_{n}$ and the automaton $\mathscr{W}_{n}$ are shown in Fig. 4.
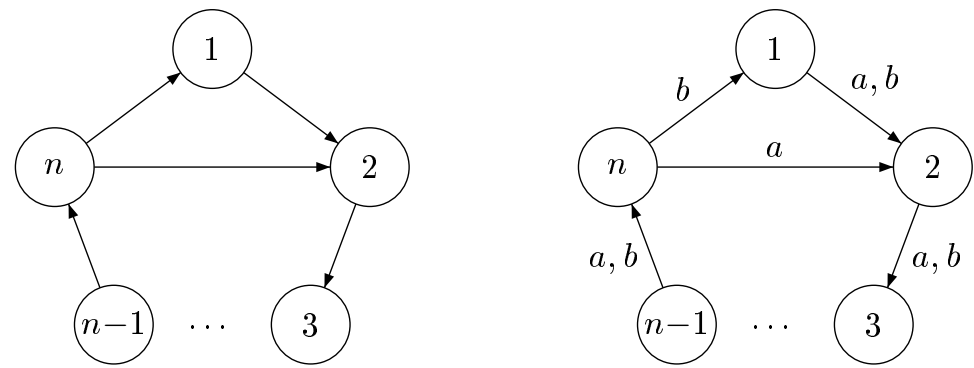

Fig. 4. The digraph $W_{n}$ and the automaton $\mathscr{W}_{n}$.

Theorem 2. The automaton $\mathscr{W}_{n}$ is synchronizing, and its reset threshold is equal to $n^{2}-3 n+3$.

Proof. It is easy to see that the word $\left(a b^{n-2}\right)^{n-2} a$ resets the automaton $\mathscr{W}_{n}$. The length of this word is equal to $(n-1)(n-2)+1=n^{2}-3 n+3$. On the other hand, Theorem 1 (b) and Proposition 2 imply that the reset threshold of $\mathscr{W}_{n}$ cannot be less than $\left((n-1)^{2}+1\right)-(n-1)=n^{2}-3 n+3$.

The series $\mathscr{W}_{n}$ was discovered by the first author in 2008. His original proof of Theorem 2 relied on a gametheoretic technique from [4] and was rather difficult.

Now we show that automata in the series $\mathscr{C}_{n}$ are also tightly related to digraphs in the series $W_{n}$, though the relation is less obvious. Note that even though the automata $\mathscr{C}_{n}$ have been known for about 50 years and have 
been rediscovered several times, to the best of our knowledge, their relationship to digraphs in the series $W_{n}$ has not been observed previously.
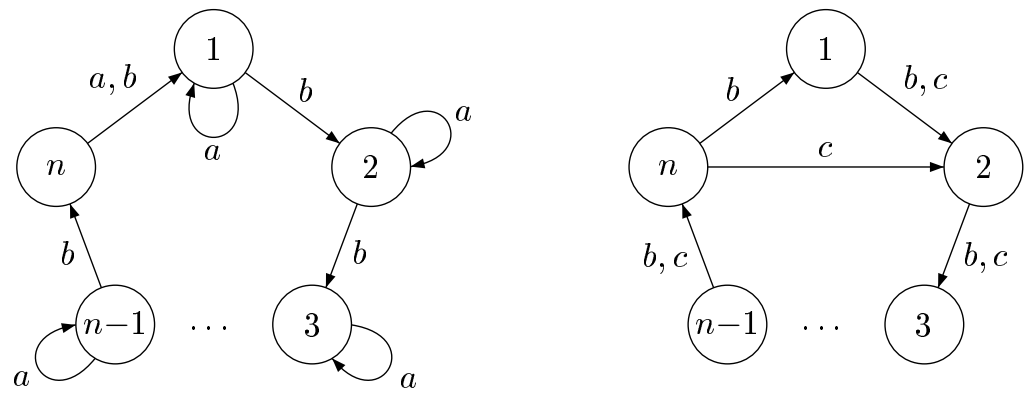

Fig. 5. The automaton $\mathscr{C}_{n}$ and the automaton defined by the action of the words $b$ and $c=a b$.

We give a new simple proof of Černý's classical result.

Theorem 3 ([9, Lemma 1]). The automaton $\mathscr{C}_{n}$ is synchronizing, and its reset threshold is equal to $(n-1)^{2}$.

Proof. It is easy to see that the word $\left(a b^{n-1}\right)^{n-2} a$ resets the automaton $\mathscr{C}_{n}$. The length of this word is equal to $n(n-2)+1=(n-1)^{2}$.

Now we invoke the following observation which will be used in some other proofs as well.

Proposition 3. Let $\mathscr{A}=\langle Q,\{a, b\}, \delta\rangle$ be a synchronizing $n$-automaton with reset threshold $t$ in which the letter a fixes all states but one and the letter $b$ acts as a permutation of the set $Q$. Consider the automaton $\mathscr{B}=\langle Q,\{b, c\}, \zeta\rangle$ in which $\zeta(q, b)=\delta(q, b)$ and $\zeta(q, c)=\delta(q, a b)$ for all $q \in Q$. Then the automaton $\mathscr{B}$ is synchronizing, and its reset threshold does not exceed $t-n+2$.

Proof. Let $w$ be a reset word of length $t$ for the automaton $\mathscr{A}$. Since the letter $b$ acts as a permutation of the set $Q$, the word $w$ cannot end with $b$, for otherwise we could obtain a shorter reset word by removing the last letter of $w$. Thus $w=w^{\prime} a$ for some word $w^{\prime} \in\{a, b\}^{*}$. Let $q_{1} \in Q$ be the unique state that is not fixed by the letter $a$, and let $q_{2}=\delta\left(q_{1}, a\right)$. The minimality of the length of the word $w$ implies that the image of the set $Q$ under the action of the word $w^{\prime}$ is equal to $\left\{q_{1}, q_{2}\right\}$.

Since the word $a^{2}$ acts on $Q$ in the same way as the letter $a$, this word cannot occur in $w$ as a factor, for otherwise we could obtain a shorter reset word by replacing the occurrence of $a^{2}$ in $w$ by $a$. Therefore, each occurrence of $a$ in $w$, except the last one, is followed by an occurrence of the letter $b$. Hence the word $w^{\prime}$ can be written as a word in the generators $b$ and $a b$. Now we replace each occurrence of the factor $a b$ in $w^{\prime}$ by an occurrence of the letter $c$, so that we rewrite $w^{\prime}$ into a word $v$ over the alphabet $\{b, c\}$. Since the words $w^{\prime}$ and $v$ act on the set $Q$ in the same way, $v c$ is a reset word for the automaton $\mathscr{B}$. Thus $\mathscr{B}$ is a synchronizing automaton; let $s$ be its reset threshold.

Since $b$ only permutes the states and each application of $c$ can send to one state only one pair of states, the word $v c$ that sends all states to a single state must contain at least $n-1$ occurrences of $c$. The length of $v$ as a word over $\{b, c\}$ is not less than $s-1$, and $v$ contains at least $n-2$ occurrences of $c$. Each occurrence of $c$ in $v$ corresponds to an occurrence of the factor $a b$ in $w^{\prime}$, whence we conclude that the word $w^{\prime}$ has length at least $(s-1)+(n-2)$. Since the length of the word $w=w^{\prime} a$ is equal to $t$, we have $t-1 \geq(s-1)+(n-2)$, whence $s \leq t-n+2$.

Observe that in what follows we will often use modifications of a given automaton $\mathscr{A}$ in the flavor of Proposition 3. In such modifications, we consider a new automaton on the same set of states but with input letters $c_{1}$ and $c_{2}$ whose actions are defined by the actions of some words $w_{1}$ and $w_{2}$, respectively, in the automaton $\mathscr{A}$. Slightly abusing terminology, we refer to the automaton arising this way as the automaton defined by the actions of the words $c_{1}=w_{1}$ and $c_{2}=w_{2}$.

We return to the proof of Theorem 3 . It is easy to see that for the automaton $\mathscr{C}_{n}$, the automaton defined by the actions of the words $b$ and $c=a b$ is isomorphic to the automaton $\mathscr{W}_{n}$, see Fig. 5 (right). By Theorem 2 , the reset threshold of $\mathscr{W}_{n}$ is $n^{2}-3 n+3$. Applying Proposition 3 , we conclude that the reset threshold of $\mathscr{C}_{n}$ cannot be less than $\left(n^{2}-3 n+3\right)+(n-2)=n^{2}-2 n+1=(n-1)^{2}$. 
The next series in the family of automata related to the digraph $W_{n}$ consists of the automata $\mathscr{E}_{n}=\langle\{1,2, \ldots, n\},\{a, b\}, \delta\rangle$ where the letters $a$ and $b$ act as follows:

$$
\delta(i, a)=\left\{\begin{array}{ll}
2 & \text { if } i=1, \\
3 & \text { if } i=2, \\
i & \text { if } i>2 ;
\end{array} \quad \delta(i, b)= \begin{cases}i+1 & \text { if } i<n, \\
1 & \text { if } i=n .\end{cases}\right.
$$

The automaton $\mathscr{E}_{n}$ is shown in Fig. 6 (left).
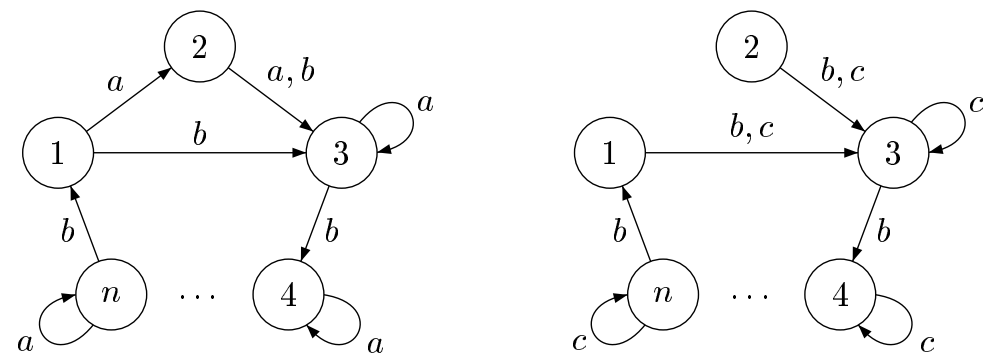

Fig. 6. The automaton $\mathscr{E}_{n}$ and the automaton defined by the actions of the words $b$ and $c=a a$.

Theorem 4. The automaton $\mathscr{E}_{n}$ is synchronizing, and its reset threshold is equal to $n^{2}-3 n+2$.

Proof. It is easy to verify that $\left(a^{2} b^{n-2}\right)^{n-3} a^{2}$ is a reset word for the automaton $\mathscr{E}_{n}$. The length of this word is equal to $n(n-3)+2=n^{2}-3 n+2$.

Now let $w$ be a reset word of minimum length for $\mathscr{E}_{n}$. We notice that in $\mathscr{E}_{n}$ the words $b a b$ and $b^{2}$ act in the same way, and so do the words $a^{3}$ and $a^{2}$. Therefore, neither $b a b$ nor $a^{3}$ can occur in the word $w$ as a factor. Besides that, $w$ cannot start with $a b$. Indeed, the image of the set of all states under the action of the word $a b$ is equal to $\{1,3, \ldots, n\}$ and thus coincides with the image of the letter $b$. Therefore, would the word $w$ start with $a b$, we could obtain a shorter reset word by replacing $a b$ by $b$. Finally, $w$ cannot end with $b a$. Indeed, if $w=w^{\prime} a$, then the minimality of $w$ implies that the image of the set of all states under the action of the word $w^{\prime}$ is equal to $\{2,3\}$. This set, however, is not contained in the image of the letter $b$, whence $w^{\prime}$ cannot end with $b$. Thus every occurrence of the letter $a$ in the word $w$ happens within the factor $a^{2}$, and no occurrences of these factors in $w$ can overlap.

Let $c=a^{2}$; then the word $w$ can be rewritten into a word $v$ over the alphabet $\{b, c\}$. The actions of $b$ and $c$ on the set $\{1,2, \ldots, n\}$ define the automaton shown in Fig. 6 (right). Since the words $w$ and $v$ act on $\{1,2, \ldots, n\}$ in the same way, $v$ is a reset word for this automaton, and hence for its subautomaton on the set $\{1,3, \ldots, n\}$. It is easy to see that the latter subautomaton is isomorphic to the automaton $\mathscr{C}_{n-1}$. By Theorem 3 , the length of $v$ as a word over $\{b, c\}$ is at least $(n-2)^{2}$ and $v$ contains at least $n-2$ occurrences of $c$. Since every occurrence of $c$ in $v$ corresponds to an occurrence of the factor $a^{2}$ in $w$, we conclude that the length of the word $w$ is not less than $(n-2)^{2}+(n-2)=n^{2}-3 n+2$.
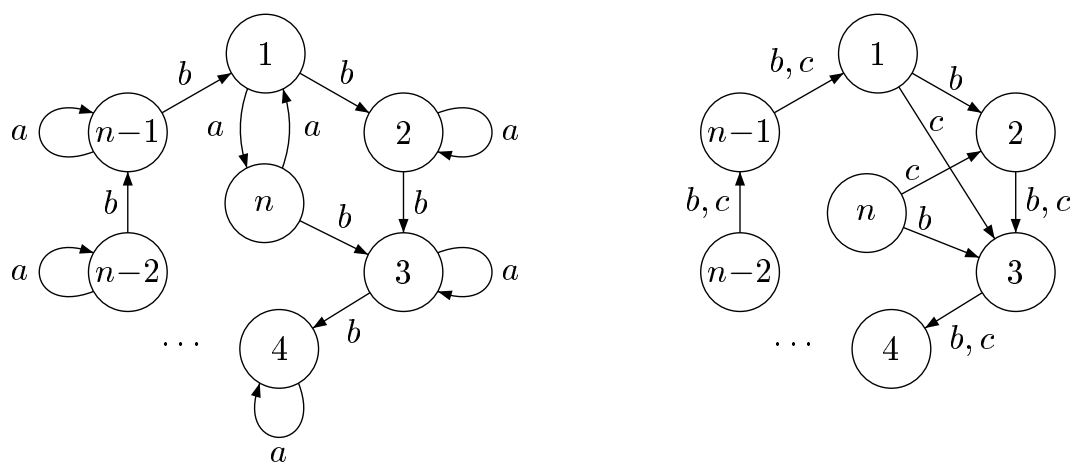

Fig. 7. The automaton $\mathscr{H}_{n}$ and the automaton defined by the actions of the words $b$ and $c=a b$.

The proof of Theorem 4 shows that the automaton $\mathscr{E}_{n}$ arises from one of the "trivial" modifications of the automaton $\mathscr{C}_{n-1}$ that we discussed in Sec. 4.1. The last series of slowly synchronizing automata from the family 
of automata related to the digraph $W_{n}$ arises from a similar modification of the automaton $\mathscr{W}_{n-1}$. The series consists of the automata $\mathscr{H}_{n}=\langle\{1,2, \ldots, n\},\{a, b\}, \delta\rangle$ where the letters $a$ and $b$ act as follows:

$$
\delta(i, a)=\left\{\begin{array}{ll}
n & \text { if } i=1, \\
i & \text { if } 1<i<n, \\
1 & \text { if } i=n ;
\end{array} \quad \delta(i, b)= \begin{cases}i+1 & \text { if } i<n-1, \\
1 & \text { if } i=n-1, \\
3 & \text { if } i=n .\end{cases}\right.
$$

The automaton $\mathscr{H}_{n}$ is shown in Fig. 7 (left).

Theorem 5. The automaton $\mathscr{H}_{n}$ is synchronizing, and its reset threshold is equal to $n^{2}-4 n+6$.

Proof. It is easy to check that the word $b\left(a b^{n-2}\right)^{n-3} a b$ resets the automaton $\mathscr{H}_{n}$. The length of this word is equal to $1+(n-1)(n-3)+2=n^{2}-4 n+6$.

Now let $w$ be a reset word of minimum length for $\mathscr{H}_{n}$. Since the word $a^{2}$ acts in $\mathscr{H}_{n}$ as the identity transformation, it cannot occur as a factor in $w$. Besides that, the word $w$ neither starts nor ends with the letter $a$, because this letter acts as a permutation of the state set of $\mathscr{H}_{n}$.

Let $c=a b$; then the word $w$ can be rewritten into a word $v$ over the alphabet $\{b, c\}$. The actions of $b$ and $c$ on the set $\{1,2, \ldots, n\}$ define the automaton shown in Fig. 7 (right). Since the words $w$ and $v$ act on $\{1,2, \ldots, n\}$ in the same way, $v$ is a reset word for this automaton. We have noticed that the word $w$ starts with the letter $b$, hence so does the word $v$. If we write $v=b v^{\prime}$ for some $v^{\prime} \in\{b, c\}^{*}$, then it is easy to see that $v^{\prime}$ is a reset word for the subautomaton on the set $\{1,2, \ldots, n-1\}$. Since this subautomaton is isomorphic to the automaton $\mathscr{W}_{n-1}$, Theorem 2 implies that the length of $v^{\prime}$ as a word over $\{b, c\}$ is at least $(n-1)^{2}-3(n-1)+3$. Besides that, $v^{\prime}$ contains at least $n-2$ occurrences of $c$, because $b$ only permutes the states and each application of $c$ can send only one pair of states to a single state. Since every occurrence of $c$ in $v^{\prime}$ corresponds to an occurrence of the factor $a b$ in $w$, we can conclude that the length of the word $w$ is not less than $1+\left((n-1)^{2}-3(n-1)+3\right)+(n-2)=n^{2}-4 n+6$.

4.3. Automata related to digraphs of the series $D_{n}$. The digraph $D_{n}$ is the $n$-digraph with exponent $(n-1)^{2}$ that corresponds to the second matrix in (1). It can be obtained from the digraph $W_{n}$ by adding the edge $(n-1,1)$. It is easy to see that, up to isomorphism and renaming of letters, there exist exactly two colorings of the digraph $D_{n}$ with two letters. Figure 8 shows the digraph $D_{n}$ and two its colorings, the automata $\mathscr{D}_{n}^{\prime}$ and $\mathscr{D}_{n}^{\prime \prime}$.
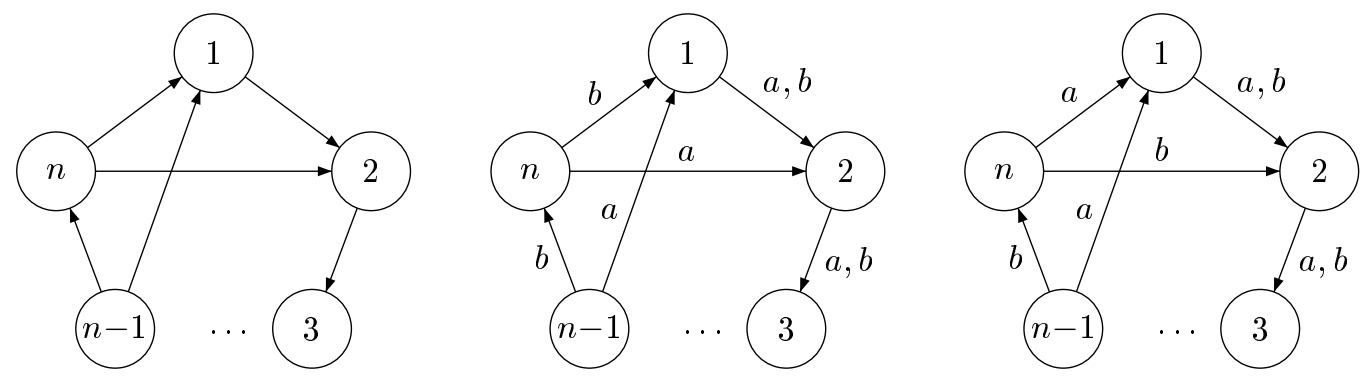

Fig. 8. The digraph $D_{n}$ and its colorings $\mathscr{D}_{n}^{\prime}$ and $\mathscr{D}_{n}^{\prime \prime}$.

Theorem 6. The automata $\mathscr{D}_{n}^{\prime}$ and $\mathscr{D}_{n}^{\prime \prime}$ are synchronizing, and their reset thresholds are equal to $n^{2}-3 n+4$ and $n^{2}-3 n+2$, respectively.

Proof. It is not hard to verify that $\left(a b^{n-2}\right)^{n-2} b a$ is a reset word for the automaton $\mathscr{D}_{n}^{\prime}$ and $\left(b a^{n-1}\right)^{n-3} b a$ is a reset word for the automaton $\mathscr{D}_{n}^{\prime \prime}$. The lengths of these words are equal to $(n-1)(n-2)+2=n^{2}-3 n+4$ and $n(n-3)+2=n^{2}-3 n+2$, respectively.

Theorem 1 (b) and Proposition 2 imply that the reset threshold for the colorings of the digraph $D_{n}$ cannot be less than $(n-1)^{2}-(n-1)=n^{2}-3 n+2$. This proves our theorem for the automaton $\mathscr{D}_{n}^{\prime \prime}$.

Now consider the automaton $\mathscr{D}_{n}^{\prime}$. Here we will make use of the following elementary result.

Lemma 1 ([17, Theorem 2.1.1]). If $k, \ell$ are relatively prime positive integers, then $k \ell-k-\ell$ is the largest integer that is not expressible as a nonnegative integer combination of $k$ and $\ell$. 
Let $w$ be a reset word of minimum length for the automaton $\mathscr{D}_{n}^{\prime}$. Since 2 is the unique state in $\mathscr{D}_{n}^{\prime}$ that is a common end of two different edges with the same label, the minimality of $w$ implies that $w$ sends all states of the automaton to 2. Assume that the length of $w$ is equal to $n^{2}-3 n+2$. Then the digraph $D_{n}$ has a directed path of this length from 1 to 2 . There is a unique edge starting at 1 , namely $(1,2)$, hence the path consists of this edge followed by a directed cycle of length $n^{2}-3 n+1$. The digraph $D_{n}$ has exactly three simple directed cycles: one of length $n$ and two of length $n-1$. Every directed cycle consists of simple directed cycles, whence the number $n^{2}-3 n+1$ (as the length of a directed cycle in $D_{n}$ ) must be a nonnegative integer combination of the numbers $n$ and $n-1$ (the lengths of simple directed cycles). However, this is impossible by Lemma 1 , since $n^{2}-3 n+1=n(n-1)-n-(n-1)$.

Now suppose that the length of $w$ is equal to $n^{2}-3 n+3$. Then the digraph $D_{n}$ has a directed path of this length from $n-1$ to 2 . Since $b$ acts as a permutation of the state set of the automaton $\mathscr{D}_{n}^{\prime}$, the word $w$ starts with the letter $a$. The state $n-1$ under the action of $a$ goes to the state 1 . Therefore, $D_{n}$ has also a directed path of length $n^{2}-3 n+2$ from 1 to 2 , but in the previous paragraph we have shown that this is impossible. Thus the length of $w$ cannot be less than $n^{2}-3 n+4$.

The series $\mathscr{D}_{n}^{\prime}$ is of interest, because for $n>6$ the automata of this series have the largest reset threshold among all known automata except those from the Cerný series $\mathscr{C}_{n}$, as well as the largest reset threshold among all known automata without loops. The series $\mathscr{D}_{n}^{\prime \prime}$ also possesses an extremal property: the automata from this series have the largest reset threshold among all known automata in which no letter acts as a permutation of the state set.

There is one further series of slowly synchronizing automata related to the digraphs $D_{n}$; it consists of $n$ automata with reset threshold $n^{2}-4 n+6$. We do not present it here, since one series with the same parameters has already been described above, see Theorem 5 .

4.4. Automata related to digraphs of the series $V_{n}$. The digraph $V_{n}$ is the $n$-digraph corresponding to the first matrix in (2). If we denote the vertices of $V_{n}$ by $1,2, \ldots, n$, then its edges are $(n, 1),(n, 3)$, and $(i, i+1)$ for $i=1, \ldots, n-1$. The digraph $V_{n}$ is primitive only if $n$ is odd, and in this case its exponent is equal to $n^{2}-3 n+4$. The digraphs of the series $V_{n}$ give rise to the family of automata $\mathscr{F}_{n}=\langle\{1,2, \ldots, n\},\{a, b\}, \delta\rangle$ in which the letters $a$ and $b$ act as follows:

$$
\delta(i, a)=\left\{\begin{array}{ll}
i & \text { if } i<n, \\
2 & \text { if } i=n ;
\end{array} \quad \delta(i, b)= \begin{cases}i+1 & \text { if } i<n, \\
1 & \text { if } i=n .\end{cases}\right.
$$

The automaton $\mathscr{F}_{n}$ is shown in Fig. 9 (left).
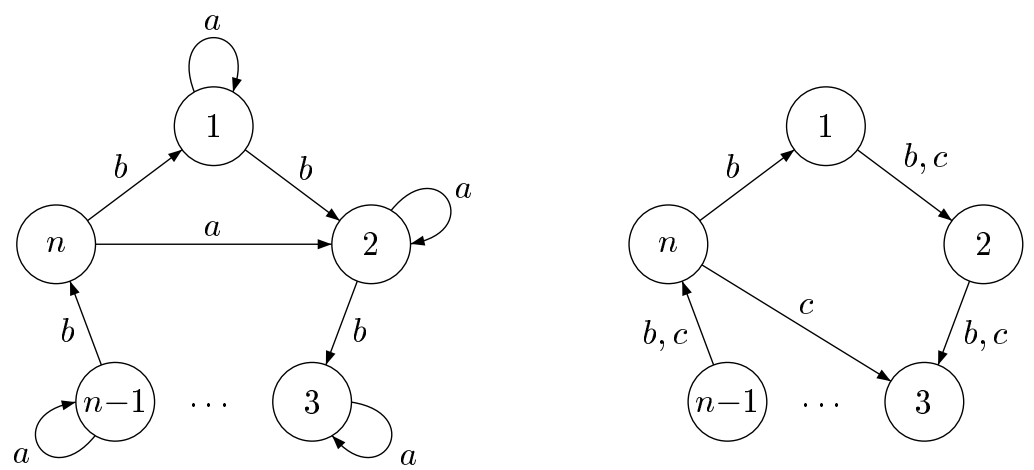

Fig. 9. The automaton $\mathscr{F}_{n}$ and the automaton defined by the actions of the words $b$ and $c=a b$.

Theorem 7. For odd $n>3$, the automaton $\mathscr{F}_{n}$ is synchronizing and its reset threshold is equal to $n^{2}-3 n+3$.

Proof. It can be easily verified that for every odd $n>3$, the word $\left(a b^{n-2}\right)^{n-2} a$ is a reset word for $\mathscr{F}_{n}$. The length of this word is $(n-1)(n-2)+1=n^{2}-3 n+3$.

Clearly, the automaton $\mathscr{F}_{n}$ satisfies the condition of Proposition 3 . The actions of the words $b$ and $c=a b$ on the set $\{1,2, \ldots, n\}$ define the automaton shown in Fig. 9 (right); we denote this automaton by $\mathscr{V}$. It is easy to see that the automaton $\mathscr{V}$ is isomorphic to a coloring of the digraph $V_{n}$. Theorem 1 (d) and Proposition 2 imply that the reset threshold for colorings of the digraph $V_{n}$ cannot be less than $\left(n^{2}-3 n+4\right)-(n-1)=n^{2}-4 n+5$. 
Applying Proposition 3, we conclude that the reset threshold for $\mathscr{F}_{n}$ cannot be less than $\left(n^{2}-4 n+5\right)+(n-2)=$ $n^{2}-3 n+3$.

4.5. Automata related to digraphs of the series $R_{n}$. The digraph $R_{n}$ is the $n$-digraph corresponding to the second matrix in (2). One obtains it from the digraph $V_{n}$ by adding the edge $(n-1,2)$. The digraph $R_{n}$ is primitive only if $n$ is odd, and in this case its exponent is equal to $n^{2}-3 n+3$. The digraphs of the series $R_{n}$ give rise to the family of automata $\mathscr{B}_{n}=\langle\{1,2, \ldots, n\},\{a, b\}, \delta\rangle$ in which the letters $a$ and $b$ act as follows:

$$
\delta(i, a)=\left\{\begin{array}{ll}
i & \text { if } i<n-1, \\
1 & \text { if } i=n-1, \\
2 & \text { if } i=n ;
\end{array} \quad \delta(i, b)= \begin{cases}i+1 & \text { if } i<n, \\
1 & \text { if } i=n .\end{cases}\right.
$$

The automaton $\mathscr{B}_{n}$ is shown in Fig. 10 (left).
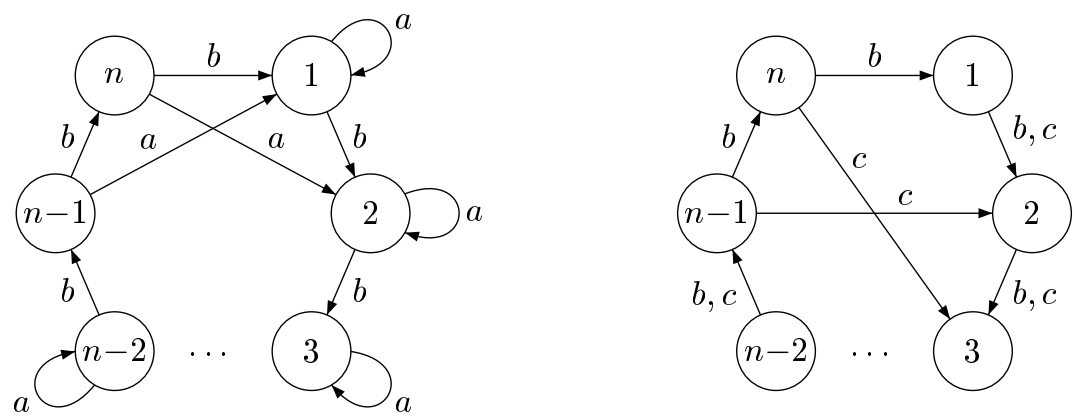

Fig. 10. The automaton $\mathscr{B}_{n}$ and the automaton defined by the actions of the words $b$ and $c=a b$.

The series $\mathscr{B}_{n}$ (for odd $n>3$ ) was described in [4], and up to recently it remained the only infinite series of slowly synchronizing automata with two input letters in the literature besides the Cerný series. The fact that the series $\mathscr{B}_{n}$ is related to digraphs from the series $R_{n}$ has not been reported earlier.

The next theorem was the main result of [4], where it was proved by a game-theoretic method. Here we present a completely elementary proof similar to the proofs of Theorems 3 and 7 .

Theorem 8 ([4, Theorem 1.1]). If $n>3$ is odd, then the automaton $\mathscr{B}_{n}$ is synchronizing and its reset threshold is equal to $n^{2}-3 n+2$.

Proof. For every odd $n>3$, the word $\left(a b^{n-2}\right)^{\frac{n-3}{2}} a b^{n-3}\left(a b^{n-2}\right)^{\frac{n-3}{2}} a$ is easily seen to be a reset word for the automaton $\mathscr{B}_{n}$. The length of this word is equal to $(n-1) \frac{n-3}{2}+n-2+(n-1) \frac{n-3}{2}+1=n^{2}-3 n+2$.

Let $w$ be a reset word of minimum length for the automaton $\mathscr{B}_{n}$, and let $t$ be the length of $w$. Since the letter $b$ acts as a permutation of the state set, the word $w$ neither starts nor ends with $b$. In particular, $w=w^{\prime} a$ for some word $w^{\prime} \in\{a, b\}^{*}$. The minimality of the word $w$ implies that the image of the state set under the action of the word $w^{\prime}$ is equal to either $\{1, n-1\}$ or $\{2, n\}$.

Since the word $a^{2}$ acts in $\mathscr{B}_{n}$ in the same way as the letter $a$, this word cannot occur in $w$ as a factor. Further, the word $b^{n}$ acts in $\mathscr{B}_{n}$ as the identity transformation, and hence it also cannot occur as a factor in a reset word of minimum length. Thus we conclude that $w=a b^{k_{1}} a b^{k_{2}} a \cdots a b^{k_{m}} a$ where $1 \leq k_{1}, k_{2}, \ldots, k_{m} \leq n-1$.

Let $c=a b$. Then the word $w^{\prime}$ and the word $v=c b^{k_{1}-1} c b^{k_{2}-1} c \cdots c b^{k_{m}-1}$ act on the set $\{1,2, \ldots, n\}$ in the same way. Therefore, the word $v c$ is a reset word for the automaton $\mathscr{R}$ defined by the actions of the words $b$ and $c=a b$ and shown in Fig. 10 (right). It is clear that the automaton $\mathscr{R}$ is isomorphic to a coloring of the digraph $R_{n}$. Theorem 1 (d) and Proposition 2 imply that the reset threshold for colorings of the digraph $R_{n}$ cannot be less than $\left(n^{2}-3 n+3\right)-(n-1)=n^{2}-4 n+4$, whence the length of $v$ as a word over $\{b, c\}$, that is, $\sum_{i=1}^{m} k_{i}$, is not less than $n^{2}-4 n+3$. Since $k_{i} \leq n-1$ for all $i=1, \ldots, m$, we have

$$
m(n-1) \geq \sum_{i=1}^{m} k_{i} \geq n^{2}-4 n+3=(n-3)(n-1),
$$

whence $m \geq n-3$. The equality $m=n-3$ is only possible if all inequalities in (5) are equalities, that is, if $k_{i}=n-1$ for all $i=1, \ldots, m$. In this case, $v c=\left(c b^{n-2}\right)^{n-3} c$, but this word is not a reset word for $\mathscr{R}$, since, as is easy to see, it permutes the states 2 and 3 . Hence $m \geq n-2$. 
Since every occurrence of $c$ in $v$ corresponds to an occurrence of the factor $a b$ in $w^{\prime}$, we conclude that the length of $w^{\prime}$ is at least $\left(n^{2}-4 n+3\right)+(n-2)=n^{2}-3 n+1$, whence the length of $w$ is at least $n^{2}-3 n+2$.

4.6. Automata related to digraphs of the series $G_{n}$. The digraph $G_{n}$ is the $n$-digraph corresponding to the second matrix in (3). One obtains it from the digraph $V_{n}$ by adding the edge $(n-2,1)$. The digraph $G_{n}$ is primitive only if $n$ is odd, and in this case its exponent is equal to $n^{2}-3 n+2$. Figure 11 shows the automaton $\mathscr{G}_{n}$, which is one of the possible colorings of the digraph $G_{n}$. This series is interesting for us, because for odd $n$ its automata attain the maximum observed "continental" value of reset threshold.

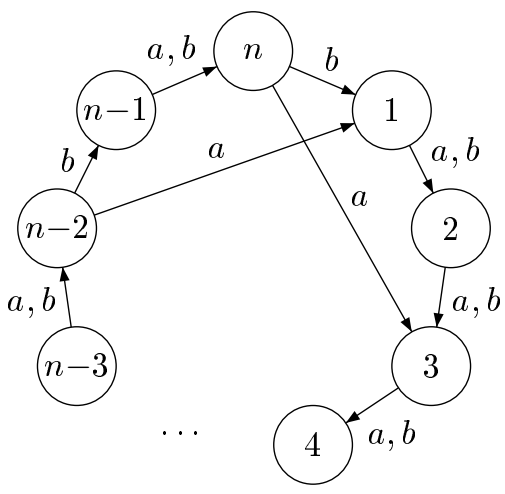

Fig. 11. The automaton $\mathscr{G}_{n}$.

Theorem 9. For odd $n>3$, the automaton $\mathscr{G}_{n}$ is synchronizing and its reset threshold is equal to $n^{2}-4 n+7$.

Proof. It is easy to see that for odd $n>3$, the word $a^{2}\left(b a b a^{n-3}\right)^{n-4} b a b a^{2}$ is a reset word for the automaton $\mathscr{G}_{n}$. The length of this word is equal to $2+n(n-4)+5=n^{2}-4 n+7$.

The following arguments are quite similar to the ones from the proof of Theorem 6 . Theorem $1(\mathrm{~d})$ and Proposition 2 imply that the reset threshold for colorings of the digraph $G_{n}$ cannot be less than $\left(n^{2}-3 n+2\right)-$ $(n-1)=n^{2}-4 n+3$.

Now let $w$ be a reset word of minimum length for the automaton $\mathscr{G}_{n}$. Since 3 is the unique state in $\mathscr{G}_{n}$ that is a common end of two different edges with the same label, the minimality of $w$ implies that $w$ sends all states of the automaton to 3. Assume that the length of $w$ is equal to $n^{2}-4 n+3$. Then the digraph $G_{n}$ has a directed path of this length from 2 to 3 . There is a unique edge starting at 2 , namely $(2,3)$, hence the path consists of this edge followed by a directed cycle of length $n^{2}-4 n+2$. The digraph $G_{n}$ has exactly three simple directed cycles: one of length $n$ and two of length $n-2$. Observe that $n$ and $n-2$ are relatively prime, since $n$ is odd. Every directed cycle consists of simple directed cycles, whence the number $n^{2}-4 n+2$ (as the length of a directed cycle in $G_{n}$ ) must be a nonnegative integer combination of the numbers $n$ and $n-2$ (the lengths of simple directed cycles). However, this is impossible by Lemma 1 , since $n^{2}-4 n+2=n(n-2)-n-(n-2)$.

Assume that the length of $w$ is equal to $n^{2}-4 n+4$. Then the digraph $G_{n}$ has a directed path of this length from 1 to 3 . There is a unique edge starting at 1 , namely $(1,2)$, hence the path consists of this edge followed by a directed path of length $n^{2}-4 n+3$ from 2 to 3 . In the previous paragraph we have shown that $G_{n}$ contains no directed path from 2 to 3 with this length.

Assume that the length of $w$ is equal to $n^{2}-4 n+5$. The word $w$ sends $n-2$ to 3 . There are two edges starting at $n-2$ : the edge $(n-2,1)$ labeled by $a$ and the edge $(n-2, n-1)$ labeled by $b$. Since the letter $b$ acts as a permutation on the state set of the automaton $\mathscr{G}_{n}$, the word $w$ starts with the letter $a$. Therefore, the first edge of the directed path from $n-2$ to 3 labeled by $w$ is necessarily the edge $(n-2,1)$, and this edge is followed by a directed path of length $n^{2}-4 n+4$ from 1 to 3 . In the previous paragraph we have shown that $G_{n}$ contains no directed path from 1 to 3 with this length.

Finally, assume that the length of $w$ is equal to $n^{2}-4 n+6$. The word $w$ sends each of the states $n-3$ and $n-1$ to the state 3 . If the second letter of the word $w$ is $a$, then the directed path from $n-3$ to 3 labeled by $w$ starts with the edges $(n-3, n-2)$ and $(n-2,1)$ followed by a directed path of length $n^{2}-4 n+4$ from 1 to 3 , and such a path is impossible. If the second letter of the word $w$ is $b$, then the directed path from $n-1$ to 3 
labeled by $w$ starts with the edges $(n-1, n)$ and $(n, 1)$, which again must be followed by an impossible directed path of length $n^{2}-4 n+4$ from 1 to 3 .

Thus we have proved that the reset threshold of the automaton $\mathscr{G}_{n}$ cannot be less than $n^{2}-4 n+7$.

\section{Discussion AND NeW CONJECTURES}

5.1. Two conjectures. The constructions and results presented in Sec. 4 show that the interconnections between reset thresholds of automata with two input letters and exponents of primitive digraphs are sufficiently tight. This conclusion is also supported by recent results of the third author [12]. We believe that these interconnections deserve further study. In order to make the future investigations more concrete, we formulate a very general conjecture in the flavor of Theorem 1. This conjecture is a strengthening of the Cerný conjecture for the case of automata with two input letters and agrees with all theoretical and experimental results that we are aware of.

Conjecture 1. (a) (The Černý conjecture). The reset threshold of every synchronizing n-automaton with two input letters does not exceed $(n-1)^{2}$.

(b) If $n>6$, then, up to isomorphism, there exists exactly one synchronizing n-automaton with two input letters and reset threshold $(n-1)^{2}$, namely, the automaton $\mathscr{C}_{n}$.

(c) If $n>6$, then there exists no synchronizing n-automaton with two input letters whose reset threshold is greater than $n^{2}-3 n+4$ but less than $(n-1)^{2}$.

(d) If $n>7$ and $n$ is odd, then, up to isomorphism, there exists exactly one synchronizing n-automaton with two input letters and reset threshold $n^{2}-3 n+4$, namely, the automaton $\mathscr{D}_{n}^{\prime}$; exactly two synchronizing $n$-automata with two input letters and reset threshold $n^{2}-3 n+3$, namely, the automata $\mathscr{W}_{n}$ and $\mathscr{F}_{n}$; and exactly three synchronizing n-automata with two input letters and reset threshold $n^{2}-3 n+2$, namely, the automata $\mathscr{E}_{n}$, $\mathscr{D}_{n}^{\prime \prime}$, and $\mathscr{B}_{n}$. There exists no synchronizing n-automaton with two input letters whose reset threshold is greater than $n^{2}-4 n+7$ but less than $n^{2}-3 n+2$.

(e) If $n>8$ and $n$ is even, then, up to isomorphism, there exists exactly one synchronizing n-automaton with two input letters and reset threshold $n^{2}-3 n+4$, namely, the automaton $\mathscr{D}_{n}^{\prime}$; exactly one synchronizing $n$-automaton with two input letters and reset threshold $n^{2}-3 n+3$, namely, the automaton $\mathscr{W}_{n}$; and exactly two synchronizing n-automata with two input letters and reset threshold $n^{2}-3 n+2$, namely, the automata $\mathscr{E}_{n}$ and $\mathscr{D}_{n}^{\prime \prime}$. There exists no synchronizing n-automaton with two input letters whose reset threshold is greater than $n^{2}-4 n+6$ but less than $n^{2}-3 n+2$.

We also formulate a more special conjecture, which can be treated as a quantitative form of the road coloring conjecture mentioned in Sec. 3. Since now we know that every primitive digraph has a synchronizing coloring [24], the notion of reset threshold can be naturally extended to primitive digraphs. Namely, we say that the reset threshold of a primitive digraph is the minimum length of reset words for all synchronizing colorings of the digraph. This immediately leads to the question of how the reset threshold of a primitive digraph depends on the number of vertices.

Note that the digraphs of slowly synchronizing automata may admit colorings with small reset thresholds. Figure 2 illustrates this remark: the first coloring of the digraph shown in the left is the Černý automaton $\mathscr{C}_{4}$, whose shortest reset word has length 9 , while the second coloring can be reset by the word $a^{3}$ of length 3 . In this connection, the series $W_{n}$ is of interest. In this series, each digraph has a unique (up to isomorphism) coloring. Therefore, the reset threshold of this coloring found in Theorem 2 coincides with the reset threshold of the digraph $W_{n}$ and provides a lower bound for the problem under consideration. We conjecture that this bound is in fact tight.

Conjecture 2. The reset threshold of every primitive $n$-digraph does not exceed $n^{2}-3 n+3$. If $n>3$, then, up to isomorphism, there exists exactly one primitive $n$-digraph with reset threshold $n^{2}-3 n+3$, namely, the digraph $W_{n}$.

Conjecture 2 has been presented in several talks of the second author since 2008, and some partial results towards its proof have already been published, see $[8,21]$. It is clear that Conjecture 2 can be made more precise in the flavor of Conjecture 1; for instance, it is likely that the digraph $D_{n}$ is the only (up to isomorphism) primitive $n$-digraph with reset threshold $n^{2}-3 n+2$, etc. 
5.2. The role of the alphabet size. In our experiments, we restrict ourselves to automata with two input letters. This restriction is caused by the fact that an increase in the alphabet size influences the number of automata much stronger than an increase in the number of states. Therefore, an exhaustive search through all automata with more than two input letters is far beyond our computational capacities even for automata with a modest number of states. Table 3 illustrates this fact. (The data in the table are calculated via a formula from [14].)

Table 3. The number of initially-connected automata with 2 and 3 input letters.

\begin{tabular}{|l||r|r|r|}
\hline \# of states & 7 & \multicolumn{1}{|c|}{8} & \multicolumn{1}{|c|}{9} \\
\hline 2 input letters & 256182290 & 12665445248 & 705068085303 \\
\hline 3 input letters & 500750172337212 & 572879126392178688 & 835007874759393878655 \\
\hline
\end{tabular}

Nevertheless, there are some reasons to expect that the behavior of the function we are interested in (the number of synchronizing automata with a fixed number of states as a function of the reset threshold) does not depend heavily on the alphabet size. For instance, Trahtman's experiments whose results were reported in [22,23] revealed no 7-automaton with 3 or 4 input letters and with reset threshold larger than 32 and smaller than 36 . Thus the value of the gap between the maximum and next-to-maximum possible values of the reset threshold is the same as for 7-automata with two input letters.

We mention also an observation that, as far as we know, was first made in [5]: if there exists an upper bound of the form $O\left(n^{2}\right)$ for the reset threshold of synchronizing $n$-automata with two input letters, then a bound of the same magnitude (but probably with a worse constant) exists also for the reset threshold of synchronizing $n$-automata with any fixed size of the input alphabet.

Acknowledgments. The authors gratefully acknowledge support from the Russian Foundation for Basic Research, grant 13-01-00852. V. V. Gusev was also supported by the program "Erasmus Mundus Action 2 Partnerships-Triple I."

Translated by the authors.

\section{REFERENCES}

1. R. L. Adler, L. W. Goodwyn, and B. Weiss, "Equivalence of topological Markov shifts," Israel J. Math., 27, 49-63 (1977).

2. M. Almeida, N. Moreira, and R. Reis, "Enumeration and generation with a string automata representation," Theoret. Comput. Sci., 387, 93-102 (2007).

3. D. S. Ananichev, V. V. Gusev, and M. V. Volkov, "Slowly synchronizing automata and digraphs," Lect. Notes Comput. Sci., 6281, 55-64 (2010).

4. D. S. Ananichev, M. V. Volkov, and Yu. I. Zaks, "Synchronizing automata with a letter of deficiency 2," Theoret. Comput. Sci., 376, 30-41 (2007).

5. M. Berlinkov, "Approximating the minimum length of synchronizing words is hard," Lect. Notes Comput. Sci., 6072, 37-47 (2010).

6. M. Berlinkov, "On a conjecture by Carpi and D'Alessandro," Internat. J. Found. Comput. Sci., 22, 15651576 (2011).

7. R. Brualdi and H. Ryser, Combinatorial Matrix Theory, Cambridge Univ. Press, Cambridge (1991).

8. A. Carpi and F. D'Alessandro, "On the hybrid Černý-road coloring problem and Hamiltonian paths," Lect. Notes Comput. Sci., 6224, 124-135 (2010).

9. J. Černý, "Poznámka k homogénnym eksperimentom s konečnými automatami," Matematicko-fyzikalny Časopis Slovensk. Akad. Vied, 14, No. 3, 208-216 (1964).

10. A. L. Dulmage and N. S. Mendelsohn, "The exponent of a primitive matrix," Canad. Math. Bull., 5, 241-244 (1962).

11. A. L. Dulmage and N. S. Mendelsohn, "Gaps in the exponent set of primitive matrices," Illinois J. Math., 8, 642-656 (1964).

12. V. Gusev, "Lower bounds for the length of reset words in Eulerian automata," Lect. Notes Comput. Sci., 6945, 180-190 (2011). 
13. J. Kari, "A counter example to a conjecture concerning synchronizing words in finite automata," Bull. Eur. Assoc. Theoret. Comput. Sci., 73, 146 (2001).

14. V. A. Liskovets, "The number of connected initial automata," Kibernetika, No. 3, 16-19 (1969).

15. J. Olschewski and M. Ummels, "The complexity of finding reset words in finite automata," Lect. Notes Comput. Sci., 6281, 568-579 (2010).

16. J.-E. Pin, "On two combinatorial problems arising from automata theory," Ann. Discrete Math., 17, 535-548 (1983).

17. J. L. Ramírez Alfonsín, The Diophantine Frobenius Problem, Oxford Univ. Press, Oxford (2005).

18. V. N. Sachkov and V. E. Tarakanov, Combinatorics of Nonnegative Matrices, Amer. Math. Soc., Providence, Rhode Island (2002).

19. S. Sandberg, "Homing and synchronizing sequences," Lect. Notes Comput. Sci., 3472, 5-33 (2005).

20. E. Skvortsov and E. Tipikin, "Experimental study of the shortest reset word of random automata," Lect. Notes Comput. Sci., 6807, 290-298 (2011).

21. B. Steinberg, "The Cerný conjecture for one-cluster automata with prime length cycle," Theoret. Comput. Sci., 412, 5487-5491 (2011).

22. A. N. Trahtman, "An efficient algorithm finds noticeable trends and examples concerning the Černý conjecture," Lect. Notes Comput. Sci., 4162, 789-800 (2006).

23. A. N. Trahtman, "Notable trends concerning the synchronization of graphs and automata," Electron. Notes Discrete Math., 25, 173-175 (2006).

24. A. N. Trahtman, "The road coloring problem," Israel J. Math., 172, 51-60 (2009).

25. A. N. Trahtman, "Modifying the upper bound on the length of minimal synchronizing word," Lect. Notes Comput. Sci, 6914, 173-180 (2011).

26. M. V. Volkov, "Synchronizing automata and the Černý conjecture," Lect. Notes Comput. Sci., 5196, 11-27 (2008).

27. M. V. Volkov, "Synchronizing automata preserving a chain of partial orders," Theoret. Comput. Sci., 410, 3513-3519 (2009).

28. H. Wielandt, "Unzerlegbare, nicht negative Matrizen," Math. Z., 52, 642-648 (1950). 